\title{
Chemical gastritis induced by naproxen in the absence of Helicobacter pylori infection
}

\author{
C J McCarthy, M McDermott, D Hourihane, C O'Morain
}

\begin{abstract}
Aim-To evaluate the histological changes that occur in the antral mucosa of healthy male subjects before and after one week of naproxen administration, using a chemical gastritis score according to the Helicobacter pylori status.

Methods-Nineteen male subjects (mean age 31 years) underwent two endoscopies: one before and the other after one week of naproxen treatment (1 $\mathrm{g}$ daily). Antral biopsy specimens were assessed for the presence of $H$ pylori infection and for chemical gastritis, defined as the presence of foveolar hyperplasia, muscle fibres in the lamina propria, oedema, and vasodilatation, in the absence of acute or chronic inflammatory cell infiltrate.

Results-Of the 19 subjects, eight had $H$ pylori infection. After one week of naproxen treatment, none of those with $H$ pylori infection developed chemical gastritis, while five of $11(45 \%)$ of those without $H$ pylori infection did. In the absence of $H$ pylori infection there was no evidence of inflammation, either before or after naproxen administration.

Conclusions-A different pattern of antral histological change occurs following naproxen administration. This pattern is related to the presence or absence of $H$ pylori infection, suggesting that $H$ pylori status should be determined in histological studies of subjects taking nonsteroidal anti-inflammatory drugs.

(F Clin Pathol 1995;48:61-63)
\end{abstract}

Keywords: Helicobacter pylori, naproxen, gastritis.

The evidence concerning the effects of nonsteroidal anti-inflammatory drugs (NSAIDs) on the gastric mucosa in subjects with Helicobacter pylori infection is conflicting. Some studies suggest that $H$ pylori infection is an important factor, ${ }^{1-3}$ while others do not support a role for $H$ pylori in NSAID gastropathy..$^{4-6}$ Lanza et $a l^{4}$ reported that volunteers with serological evidence of $H$ pylori infection had similar macroscopic antral mucosal changes to those without $H$ pylori infection after one week of naproxen or aspirin treatment. However, as macroscopic changes do not correlate well with the histological changes of the gastrointestinal mucosa ${ }^{7}$ the above observations need to be confirmed at the histological level. ${ }^{4}$

The histological changes that occur in the intact stomach secondary to either bile reflux, drugs, or other chemicals has been termed chemical gastritis (type $\mathrm{C}$ or reactive gastritis)..$^{89}$ This entity appears to be more prevalent in NSAID users. ${ }^{3910}$ The latter histological studies examined biopsy specimens of patients after ingestion of NSAIDs. To date, however, there have been no prospective studies assessing the changes leading to chemical gastritis before and after administration of NSAIDs. The aim of this study was to assess any histological changes occurring in the antral mucosa, using a chemical gastritis score, depending on $H$ pylori status both before and after one week of naproxen treatment.

\section{Methods}

Nineteen healthy men with a mean age of 31 years (range $25-41$ years) were studied. They had no history of peptic ulcer disease, upper gastrointestinal symptoms, and had not received NSAIDs, bismuth, or antibiotics over the preceding three months.

The study was approved by the local ethics committee and informed consent was obtained from all subjects.

Each volunteer underwent two gastroscopies: one at entry and the other eight days later. Study medication comprised naproxen, $1 \mathrm{~g}$ daily, on days 0 to 7 which was taken at the same time each day following a meal. The subjects abstained from alcohol during the study period. Each gastroscopy was performed by one endoscopist who assessed the macroscopic appearance of the mucosa using the Lanza score ${ }^{11}$ as follows: $0=$ normal mucosa with no submucosal haemorrhage; 1 = a single submucosal haemorrhage; $2=$ more than one submucosal haemorrhage; $3=$ numerous areas of submucosal haemorrhage; and $4=$ large areas of submucosal haemorrhage with active bleeding or widespread inflammation of the stomach.

At each endoscopy, two antral biopsy specimens were taken from an area of normal looking mucosa (2 $\mathrm{cm}$ from the pylorus): one for a rapid urease test (CLO test, Delta West Ltd., Bentley, Australia) to detect the presence of $H$ pylori and the second for histological assessment. Specimens for histology were fixed in $10 \%$ formalin and embedded in paraffin wax. Sections from the paraffin wax blocks were stained with haematoxylin and eosin, and a further section was stained with Warthin-Starry for the identification of $H$ pylori. Slides were examined by two pathologists. One $(\mathrm{DH})$ recorded the presence or 
absence of chronic gastritis and $H$ pylori. The second $(\mathrm{MMcD})$, unaware of the findings of the first pathologist, assessed the histological features forming the basis of the chemical gastritis score. ${ }^{8}$ These features include the presence of foveolar hyperplasia (0-3), lamina propria oedema (0-3), and vascular congestion (0-3). These scores were added to two scores which assessed acute (0-3) and chronic $(0-3)$ inflammatory cell infiltrate $(3=$ no inflammatory infiltrate; $0=$ severe inflammatory infiltrate). The highest possible score was 15. A total score of 10 or more was indicative of chemical gastritis in accordance with the observations of Dixon et al. ${ }^{8}$ Both pathologists were blind to the day on which the biopsy specimens were taken, the endoscopic appearance of the gastroduodenal mucosa at the time of endoscopy, and the result of the rapid urease test.

\section{Results}

Of the 19 subjects, eight were infected with $H$ pylori, as documented by a positive rapid urease test and the presence of $H$ pylori on antral histology. $H$ pylori status did not change following administration of naproxen for one week. Two of the $H$ pylori positive and three of the $H$ pylori negative subjects were smokers.

There was no evidence of macroscopic mucosal damage in any of the subjects at their first endoscopy. After one week of naproxen administration, the mean (SD) macroscopic score $^{11}$ was $1.6(0.5)$ in those with and 2.0 $(0.5)$ in those without $H$ pylori infection. Of the subjects, 14 of 19 (74\%) had some evidence of macroscopic inflammation after one week, six of whom were infected with $H$ pylori. Changes in the macroscopic appearance of the gastroduodenal mucosa after naproxen administration were similar in the subjects with and without $H$ pylori infection.

All of the subjects with $H$ pylori infection had mild or moderate chronic gastritis. None of these subjects had chemical gastritis. Of the $11 \mathrm{H}$ pylori negative subjects, one had chemical gastritis. The remaining subjects, with chemical gastritis scores of 10 or less and no evidence of chronic gastritis, were regarded as histologically normal. None of the subjects had concurrent chemical and chronic gastritis.

None of the $H$ pylori positive subjects ( $\mathrm{n}=$ 7 ; one subject did not have an adequate specimen for examination and was excluded from subsequent analysis) had chemical gastritis scores greater than 10 following naproxen administration. Indeed, three subjects had a lower score, reduced by one point in each case. This was because of an increase in the severity of chronic gastritis which was noted in four subjects. Three subjects had an increased chemical gastritis score (by two, four and five points, respectively) which occurred in one case despite a concurrent increase in chronic inflammation, which obviously depresses the score. The score remained unchanged in one case.

Of the $11 \mathrm{H}$ pylori negative subjects, two had a reduced chemical gastritis score, by one and two points, respectively. Six (55\%) subjects had an increased score (by one, one, three, three, four, and five points, respectively) and in five (45\%) of these cases, the resulting score now fulfilled the criteria for chemical gastritis. The score remained unchanged in three cases, none of whom had concurrent chemical and chronic gastritis.

\section{Discussion}

Previous studies have confirmed that chemical gastritis occurs in NSAID users. ${ }^{3910}$ However, this is the first report highlighting that chemical gastritis develops after just one week of treatment. The study is strengthened by its prospective design which assessed a single anti-inflammatory drug-naproxen. We also identified differences in the histological changes occurring in the antral mucosa of $H$ pylori positive and negative subjects. Those with $H$ pylori infection did not have or develop chemical gastritis, while $45 \%$ of those without $H$ pylori infection developed chemical gastritis following treatment with naproxen. Conversely, as previously reported, ${ }^{12}$ chronic gastritis was strongly associated with $H$ pylori infection and those without $H$ pylori infection did not have chronic gastritis either before or after naproxen administration.

Recently, Quinn et $a l^{10}$ reported that chronic and chemical gastritis co-exist in a subgroup of patients. While the possibility that the two conditions were distinct entities occurring concurrently was considered, the preferred explanation was that this mixed category represented an overlap of the spectrum of the two diseases. Such an overlap, however, was not observed in any of the subjects included in this study. This discrepancy is almost certainly because of the different methods used to assess chemical gastritis. In the scoring system proposed by Dixon et $a l^{8}$ the presence of an inflammatory cell infiltrate leads to a reduction in the overall chemical gastritis score. Only in very exceptional circumstances (not encountered in our relatively small study group), where the inflammatory cell infiltrate is very mild and the other measured histological features are severe, is it possible to exceed a score of 10 . Therefore, the points system makes the diagnosis of chronic and chemical gastritis almost mutually exclusive. Such an interpretation is supported by the strong association demonstrated in this study and in others ${ }^{3}$ between colonisation by $H$ pylori and chronic gastritis on the one hand and the absence of $H$ pylori and chemical gastritis on the other. At present, the importance of increases in the chemical gastritis score which fail to reach a threshold of 10 points is uncertain.

The less formal system adopted by Quinn et $a l^{10}$ where the individual histological features of reactive gastritis are still sought but no scoring system is employed, enables the pathologist to make the combined diagnosis. However, because the histological features comprising chemical gastritis are non-specific and may be seen in other gastric pathologies, ${ }^{10}$ 
such a method runs the risk of interpreting non-specific findings as chemical gastritis. ${ }^{810}$ We would propose, therefore, that the diagnosis of chemical gastritis be made only when all of the histological features, including a lack of inflammatory cell infiltrate, are present and that, unless further studies clarify the situation, types B and C gastritis remain, histologically at least, mutually exclusive.

The relation between $H$ pylori and NSAID gastropathy is similarly unclear, although there is in vitro evidence that NSAIDs are more damaging to the antral mucosa in the presence of $H$ pylori. ${ }^{13}$ In a study of 218 patients, ${ }^{3}$ of whom 174 were taking NSAIDs, there was a higher incidence of ulceration in NSAID users with than in those without $H$ pylori infection, suggesting that NSAIDs and $H$ pylori infection act synergistically. ${ }^{14}$ In this study macroscopic lesions were observed with equal frequency in both $H$ pylori positive and negative subjects. Microscopically, chemical gastritis, the pattern of gastric damage now thought to be associated with NSAID administration, was not observed in those with $H$ pylori infection. However, prevalence of chronic gastritis, a pattern strongly associated with $H$ pylori infection, was increased following administration of naproxen as observed in four of seven subjects with $H$ pylori infection. Although the numbers in this study are small, these results suggest that administration of NSAIDs increase the inflammation associated with established $H$ pylori infection, rather than that $H$ pylori infections increases NSAID gastropathy.

Macroscopic mucosal lesions were present in $14(74 \%)$ subjects following naproxen administration. There was no evidence of macroscopic inflammation before ingestion of the drug. As $H$ pylori gastritis is poorly correlated with macroscopic lesions ${ }^{71516}$ and because chemical gastritis can occur in patients taking NSAIDs, histological evaluation of the gastric mucosa is important for assessing mucosal changes in patients taking NSAIDs, who may also be infected with $H$ pylori. Macroscopic lesions are unlikely to be related to the presence of $H$ pylori infection in patients with rheumatoid arthritis who have been taking NSAIDs chronically, ${ }^{17}$ again suggesting that histological assessment of the mucosa is necessary. The macroscopic scores in this study were similar to those obtained by Lanza et $a l,{ }^{4}$ with both $H$ pylori positive and negative subjects having an increased macroscopic score; however, in this study these changes did not correlate with the histological changes observed.
In conclusion, this study has documented the pattern of histological changes occurring in the antral mucosa after one week of naproxen treatment and has recorded differences between subjects with and without $H$ pylori infection. Clearly, $H$ pylori infection should be identified and considered in all studies evaluating the effects of NSAIDs on the gastric mucosa.

1 Jones STM, Clague RB, Eldridge J, Jones DM. Serological evidence of infection with Helicobacter pylori may predic gastrointestinal intolerance to non-steroidal anti-inflammatory drug treatment of rheumatoid arthritis. $\mathrm{Br} \mathcal{F}$ Rheumatol 1991;30:16-20.

2 Upadhyay R, Howatson A, McKinlay A, Dannesh BJZ, Sturrock RD, Russell RI. Campylobacter pylori associated gastritis in patients with rheumatoid arthritis taking nonsteroidal anti-inflammatory drugs. Br $\mathcal{F}$ Rheumatol 1988 ; 27:113-16.

3 Taha AS, Nakshabendi I, Lee FD, Sturrock RD, Russell RI. Chemical gastritis and Helicobacter pylori related gastritis in patients receiving non-steroidal anti-inflammatory drugs: comparison and correlation with peptic ulceration. $f$ Clin Pathol 1992;45:135-9.

4 Lanza FL, Evans DG, Graham DY. Effect of Helicobacter pylori infection on the severity of gastroduodenal mucosal injury after the acute administration of naproxen or aspirin to normal volunteers. $\mathrm{Am} f$ Gastroenterol 1991;86:735-7.

5 Graham DY, Lidsky MD, Cox AM, Evans DJ Jr, Evans DG, Alpert $\mathrm{L}$, et al. Long-term nonsteroidal anti-inflammatory drug use and Helicobacter pylori infection. Gastroenterology 1991;100:1653-7.

6 Peterson WL, Lee E, Feldman M. Relationship between Campylobacter pylori and gastritis in healthy humans after Campylobacter pylori and gastritis in healthy humans after the administration of placebo

7 Vaira D, Holton J, Osborn J, D'Anna L, Romanos A Falzon $\mathrm{M}$, et al. Endoscopy in dyspeptic patients: is gastric mucosal biopsy useful? Am F Gastroenterol 1990 85:701-4.

8 Dixon MF, O'Connor HJ, Axon ATR, King RFJG Johnston D. Reflux gastritis: a distinct pathological entity? F Clin Pathol 1986;39:524-30.

9 Sobala GM, King RFG, Axon ATR, Dixon MF. Reflux gastritis in the intact stomach. F Clin Pathol 1990;43: 303-6.

10 Quinn CM, Bjarnason I, Price AB. Gastritis in patients on non-steroidal anti-inflammatory drugs. Histopathology 1993;23:341-8.

11 Lanza FL, Royer GL, Nelson RS. Endoscopic evaluation of the effects of aspirin, buffered aspirin, and enteric coated aspirin on the gastric and duodenal mucosa. N Engl f Med 1980;303:136-8

12 Dooley CP, McKenna D, Humphreys H, Bourke S, Kean $\mathrm{CT}$, Sweeney E, et al. Histological gastritis in duodenal ulceration: relationship to Campylobacter pylori and the effect of therapy. Am $\mathcal{F}$ Gastroenterol 1988;83:278-81.

13 Taha AS, Kelly RW, Gemmell CG, Lee FD, Russell RI The interaction between Helicobacter pylori culture filthate and indomethacin: effects on integrity of human trate and indomethacin: effects on ingegrity of human tion in vitro. Aliment Pharmacol Ther 1990;4:265-74.

14 Taha AS, Russell RI. Helicobacter pylori and non-steroidal anti-inflammatory drugs: uncomfortable partners in pepanti-inflammatory drugs: uncomforta

15 Merki HS, Meyer-Wyss B, Mazzucchelli L, Wilder-Smith CH, Ruchti C, Renner E, et al. Prevalence of macroscopic lesions in the upper GI-tract and histological gastritis in asymptomatic people. Gastroenterology 1992;102 A21.

16 Schnegg JF, Bah A, Saraga E, Armstrong D, Vouillamoz $D$, Dorta $G$, et al. Do endoscopic appearances contribute to the diagnosis of Helicobacter pylori-associated gastritis. Gastroenterology 1992;102:A161.

17 Loeb DS, Talley NJ, Ahlquist DA, Carpenter HA Zinsmeister AR. Long term use of nonsteroidal antiinflammatory drugs and gastroduodenal injury; the role inflammatory drugs and gastroduodenal injury; the rol 1899-905. 